\title{
perifèria
}

Número 14, junio 2011

www.periferia.name

\section{Antropología multi-situada y "Lifestyle Sports": Por un examen de la escalada a través de sus espacios}

\author{
Guillaume Dumont ${ }^{1}$
}

\begin{abstract}
Resumen
El objetivo de este artículo es repensar la construcción de los espacios por medio de una práctica deportiva, la escalada, realizada como un modo de vida. Desde el punto de vista de la antropología de los espacios a las concepciones más pragmáticas que surgen del trabajo de campo multi-situado, varias tradiciones han sido utilizadas para examinar el rol que ocupan los espacios de práctica en los lifestyle sports (Wheaton, 2004) modernos. Sus construcciones, apropiaciones, utilizaciones y deterioraciones, son elementos de un proceso complejo que ha sido poco desarrollado en la teoría de las "nuevas prácticas deportivas" tal como el surf, el snowboard, el skateboard y, en nuestro caso, la escalada. En efecto, si estos fenómenos han sido siempre examinados desde el punto de vista de la contracultura, también contendrán dinámicas que van más allá de una moda joven básica y que revelan fenómenos sociales y espaciales complejos. Además, mirar estas prácticas tras el vidrio del espacio permite replantear algunos elementos metodológicos propios de la práctica del trabajo etnográfico y de sus condiciones gracias a fenómenos específicos en este campo.
\end{abstract}

Palabras clave: lugar y espacio, semiótica y simbólica del espacio, lifestyle sports, antropología multi-situada.

\begin{abstract}
The aim of this paper is re-thinking the construction of space through an alternative sport, climbing, acted as a way of life by those who practise it. From the point of view of the Anthropology of space to the emergent pragmatic conception of a multisite field research, different traditions have been mobilized to examine the importance of the sites of practice in regards to modern lifestyle leisure's. The construction, appropriation, uses and degradations of the space, as elements of a complex process which have been relatively denigrated and left out in the theoretical production linked to these "news sport activities" such as surfing, snowboarding, skateboarding and, in this case, sport climbing. In fact, most of the studies dedicated to theses phenomena's focused on the counter-cultural aspects
\end{abstract}

\footnotetext{
${ }^{1}$ Enviar correspondencia a: guidumo@gmail.com
} 


\section{perifèria}

Número 14, junio 2011

www.periferia.name

without giving importance to the social and spatial dynamics, which go beyond a youth's simple and modern tendency and are actually of a much more complex nature. Furthermore, examining theses practices through the frame of the concept of space allows a reflexion on certain methodological tools of the ethnographic practice and it's conditions, due to the specificity of this kind of field.

Key words: Place and space, semiotic and symbolic of the space, lifestyle sports, multi-sited anthropology

\section{Elementos de metodología}

Los datos de este artículo se extraen de un trabajo de campo multi-sitio intensivo de 4 meses, previamente delimitado por varios trabajos de campo exploratorios durante dos años, y bajo la utilización de los instrumentos clásicos de la etnografía como la "participant observation" (Wacquant, 2004). Estos trabajos de campo han sido realizados con escaladores semi-profesionales seleccionados mediante el criterio clave de la frecuencia de práctica, como una práctica cotidiana. Estos "fanáticos", o "core members" en la teoría de la sociología del deporte (Wheaton, 2000; Beal and Wilson, 2004; Donnelly, 2006) forman una pequeña parte del grupo de los escaladores, llegando al punto de dedicar todos los aspectos de su vida a la escalada para hacer de ella su modo de vida. En efecto, desde sus trabajos a sus redes sociales, hay pocas cosas en su día a día que no estén relacionadas con esta práctica. Como lo veremos más adelante, estos grupos comparten símbolos, normas y referencias que se desarrollan en espacios de práctica territorializados donde la entrada del etnógrafo no suele ser fácil. Por eso, la utilización de la participant observation ha sido clave en la realización de la investigación, donde el etnógrafo no sólo observa sino que tiene que demostrar sus conocimientos, capacidades y comprometerse, compartir referencias y condiciones de vida.

En términos formales, el objetivo de esta investigación es describir cómo y por qué estos escaladores han adoptado la escalada como modo de vida, poniendo énfasis sobre el desarrollo y las implicaciones desde el punto de vista socio-antropológico, lo que ha permitido tratar varios temas como, por ejemplo, las relaciones sociales en estos grupos, el aprendizaje, la cuestión del gusto en general, la utilización de los espacios de práctica y una crítica de la "théorie de l'action" (Bourdieu, 1994). 


\section{perifèria}

Número 14, junio 2011

www.periferia.name

Aunque este trabajo tenía que ser descriptivo y exploratorio, su realización ha sido guiada por una serie de hipótesis formuladas durante la parte exploratoria de la investigación, sobre bases de datos recogidos y la teoría especializada, junto a un análisis de las producciones mediáticas relacionadas con la escalada. En efecto, las revistas, las películas, y sobre todo la información contenida en Internet han sido una fuente de datos importante sobre las actividades de los escaladores, mediante blogs y varias webs especializadas, antes, durante y después de la realización del trabajo de campo. A día de hoy, obviar los "subcultural media" (Wheaton and Beal, 2003) que incluyen la producción mediática propia sobre la práctica estudiada, sería dejar de lado un aspecto clave, por lo que se ha tenido en cuenta la importancia de sus actores y su carácter determinante en la elección de los sitios y tipos de práctica, lo que tiene consecuencia directa sobre la planificación del trabajo de campo.

Además, el desarrollo de lo que se puede llamar lifestyle sports, como la escalada, se ha relacionado con la producción y difusión de esas informaciones, por lo que no sólo se habla de producción sino además de la recuperación de los actores y de la industria del deporte (Beal and Wheaton, 2003; De Leseleuc, 2004; Aubel, 2005; Soulé et Walk, 2007).

Así, los sitios del trabajo de campo han sido elegidos a partir de esta exploración, así como su planificación y desarrollo. En efecto, estudiar una práctica deportiva que depende de su medio ambiente implica tener en cuenta este mismo. Ha sido necesario seguir las temporadas de escalada y la frecuentación de las zonas para elegir los sitios del trabajo de campo y el método de investigación. Por eso, el trabajo de campo ha sido realizado en España (Albarracín y Madrid) entre febrero y abril, y en Suiza (Averstaal) durante mayo y junio 2010. 


\section{perifèria}

Número 14, junio 2011

www.periferia.name

\section{Gusto y viaje: por una introducción a la práctica}

La escalada como modo de vida implica salir del cuadro de la vida cotidiana, adoptando formas de vida más marginales que desviadas, aunque este sigue siendo un debate abierto. Vivir por y para la escalada implica sacrificar aspectos de la vida, tanto a nivel físico como social. Lo que nos interesa aquí son las condiciones de vida propias de esta práctica, tanto a nivel de "espacio de vida" como la movilidad que implica la escalada. En efecto, a este nivel de práctica, estamos frente a una actividad totalmente dependiente de su medio ambiente, del sitio que permite y apoya su desarrollo. Además, factores como las condiciones meteorológicas son elementos de primera importancia en la cotidianidad de los escaladores, igual que en otros lifestyle sports. Estos factores van a tomar una importancia clave sobre varios aspectos, desde la elección de los sitios de práctica hasta los desplazamientos de una zona a otra. Por ejemplo, cuando examinamos los niveles de frecuentación de los sitios con las temporadas del año.

También, estos sitios sirven para varias funciones, por parte de los escaladores serán utilizados como espacios de vida, donde un parking puede ser utilizado como un "hábitat" con su semiótica y simbología propia del espacio. Así que, detrás de lo obvio, se revelan las características de una práctica que no sólo se considera como un deporte sino como un modo de vida.

Desde que tengo coche, iya no he parado! Que sea media hora, 20 días o tres meses a Sudáfrica, da igual. He viajado por muchos sitios, el otro día lo decía, desde Eslovaquia a San Francisco, y desde Capetown hasta Noruega. Pues todo eso, más o menos he visto miles de sitios de escalada en casi todo el mundo, se ha convertido en un rollo que casi todo lo que hago en mi vida es para poder irme a escalar a un sitio guapo [...] Y viajar desde siempre porque siempre me ha gustado, todo el grupo de colegas que tengo, somos un poco más...viajar y tal, porque me fui con 17 años a Cuenca en tren, a escalar, pues ahora que tengo 30 años fui a Sudáfrica, o el mes del año anterior a Yosemity, Bishop y...otro sitio, donde quería estar... (Davilo, 30 años, España)

De esta manera, podríamos relacionar este fenómeno con el modo de vida de los 


\section{perifèria}

Número 14, junio 2011

www.periferia.name

gitanos, población que siempre ha estado ligada con fenómenos de desplazamiento y a una vida al aire libre, aunque en la realidad son totalmente diferentes los imaginarios colectivos.

When we told to people than we gonna live in the van and travel around the year, they were like: "U living in a van...?!" And we were like "Yeah!" And there were like..."Right" and then...you could see the different types of people you're talking to....when I told to my dad he was like: "O cool...!" And he has been really interested in and you talk to other people and they are like: "Ouh, it's like being a gipsy!" Yeah, it's exactly like being a Gipsy! Fuck off, do what you like and they were like...it was interesting to see people having different perception of it, some people was fucking jealous! Wish I can just get up and go and that's like anybody can... (Leah, 20 años, Reino Unido)

Esta transformación de los espacios de práctica en espacios de vida se realiza en paralelo con la territorialización y la aparición de normas en estos espacios ${ }^{2}$, los cuales dependen de la concepción del "local", concepto que será desarrollado más adelante. Apropiarse de un espacio y acondicionarlo, implica normas y reglas, maneras de actuar, así como el dominio de un sistema de referencias.

Así que, se puede afirmar la existencia de un cierto way of thinking o "Ethos" (Stebbins, 1992) compartido por la mayoría de escaladores, Ethos que ha sido construido individual y colectivamente. De manera más concreta, este "Ethos" es, sobre ciertas formas, afirmado y reivindicado como diferente, peculiar, y específico a los escaladores como lo describe Simon:

It's like going to Cuenca and not knowing anyone but because you're a climber and somebody else is a climber, they just go... because you're climber. And I know than because you're a climber and I'm a climber it's fine (laughter) [...] And it's the way it is, it's really cool! It's like a community! Yeah it's not the same if you're a golfer you not you just gonna go...because you're a golfer I know that mean you understand and [...] but you not gonna

\footnotetext{
${ }^{2}$ Véase a De Leseleuc, 2004
} 


\section{perifèria}

Número 14, junio 2011

www.periferia.name

go like, stayed at the house of a golfer cause you play golf together, it's not the same...The people who were there, with me, also feel the same way than I do, a kind of this is not good! All the others people are just bro and we understand. And with climbing there's not da many people doing it so maybe it's the same thing. You get to the football, is like you and 40.000 other people, that's weird! (Simon, 25 años, Reino Unido)

Los espacios ocupados y los sitios de escalada visitados por el escalador fanático van a servir para esa construcción y particularmente como formas de referencias, como criterios de una actividad pasada y como parte de la construcción del gusto común por ser parte del "subcultural capital" (Thornton, 1997). Desde las características propias del sitio, es decir su acceso, su entorno, sus sitios "clave" y puntos de encuentro, hasta el carácter de los "bloques" o de las "vías", los diferentes tipos de "agarres" y, en general, el estilo de escalada, haber ido y haber escalado en una zona famosa de escalada, un "Haut-lieux" (Sayeux, 2010) forman parte integrante del "escalador fanático". Así que, es muy común ver a dos escaladores discutir durante horas sobre las características y los "agarres" de una piedra situada al otro lado del mundo, sobre las maneras de escalarla, las sensaciones experimentadas y el grado de dificultad de una "vía".

\section{Lugares y espacios: Por una antropología de los paisajes}

Los paisajes han sido estudiados en la producción antropológica como parte de un campo más amplio, utilizados como soporte más que como elemento individual (Lovell, 1998). Según Hirsch (1995) los paisajes han sido cuadros de desarrollo de la vida, elementos constitutivos de la relación entre el hombre y la naturaleza, de cualquier tipo. Si Descola (2005) nos propone una visión radicalmente diferente de esas relaciones, los procesos de construcción semiótica relacionados a los espacios de vida y la influencia bilateral entre el ser humano y la naturaleza han sido ilustrados anteriormente, tal como lo hizo la obra colectiva de Nadia Lovell (1998). En esa teoría, lo que nos interesa es la implicación semiótica y simbólica en una perspectiva que se proyecta en los individuos y sus prácticas, lo mismo que la construcción de estas en relación con el medio ambiente en el cual se desarrollan. 


\section{perifèria}

Número 14, junio 2011

www.periferia.name

En efecto, la relación de los escaladores, su medio ambiente y sitios de práctica es crucial frente a la adopción de esa práctica como modo de vida:

I thing a big part of climbing it's just being outside, in beautiful areas, and just staying in Israel which I know for a long time all the climbing areas or staying in the gym it's not enough! It's good for training or when you don't have enough money to go, but the main reason it's just being outside in beautiful places. (Gil, 24 años, Israel)

La práctica influye en el sitio y el sitio en la práctica, así que un análisis de la construcción de estos sitios y su apropiación es indispensable, además de que puede reflejar una cierta idea de la sociedad según Sayeux (2010).

Para tratar estos procesos de implicación semiótica del lugar y de la producción de los espacios, varias inflexiones teóricas nos permiten ver estos fenómenos desde diferentes ángulos determinados por los conceptos de "lugar" y "espacio". Desde las perspectivas de Michel De Certeau, Maurice Merleau-Ponty y Henri Lefebvre, se puede considerar el lugar como un espacio implicado ${ }^{3}$, aunque De Certeau no concreta la idea de un lugar asociado a la práctica porque es totalmente dependiente de la "phénomologie de l'exister au monde". Merleau-Ponty nos ofrece una visión más sencilla y práctica de esas definiciones, sin el pragmatismo de De Certeau: "Esta experiencia es relación con el mundo; en el sueño y en la percepción y por así decirlo anterior a su diferenciación, expresa la misma estructura esencial de nuestro ser como ser situado en relación con un medio ambiente, un ser situado por un deseo, indisociable de una dirección de la existencia" (Merleau-Ponty, 1985: 130). Entonces, hay tantos espacios diferentes como experiencias espaciales, el espacio es un producto individual y colectivo y, siguiendo a Lefebvre (1974), un producto social.

O man, I really love this place. The trees are beautiful, the landscape is amazing, and the rock is so good! Check this hold man, its perfect! I really love this sandstone! I really like this lifestyle, just being there...I feel like on

\footnotetext{
3 « Un espace investi » (De Certeau, 1990)
} 


\section{perifèria}

Número 14, junio 2011

www.periferia.name

another planet, this place is unique. Este sitio es mágico, te engancho. (Lorenzo, 29 años, Alemania)

El desarrollo y la producción de estas experiencias, en parte relacionado con el lugar y el espacio, supone ciertas aficiones a aquellos lugares que pueden conceptualizarse mediante los conceptos de "Locality" y "Belonging", conceptos que nos permiten igualmente introducir la noción de identidad, tanto desde el punto de vista individual como colectivo.

The local is conditioned into being, and invoked into existence, through the necessity of creating an "other" who is different from ourselves as possible, and is therefore often transformed into an highly artificial construct bounded both by pragmatic constraint of fieldwork and academic expectations. (Lovell, 1998: 5)

La memoria asociada a una identidad individual y colectiva, pasada o presente, queda ligada a la idea de lugar, tal como una entidad individual y fija con un marco espacial preciso. Cuando eso se realiza, hay que tener en cuenta el hecho ilustrado por Appadurai (1991) y definido como "deterriorialised identity", que sitúa la identidad entre varios sitios, más que en un único sitio. Este proceso entra directamente en la construcción y la repartición de referencias, como ya ha sido tratado antes, referencias de la identidad de los escaladores. En efecto, el hecho de vivir en un sitio de escalada implica dominar sus normas y códigos, que pueden ser traslados a otros sitios y que, un vez más, forman parte de la identidad, permitiendo al escalador adoptar el comportamiento adecuado al sitio donde está escalando.

Compartida entre lugares, la identidad no es múltiple; su multiplicidad se encuentra en su construcción y su transformación (Donnelly and Young, 1988), mucho más en nuestro caso, donde está caracterizada por la noción de movimiento. Entonces, si la identidad individual es multi-local, la identidad colectiva necesita una investigación más profunda, en relación con los procesos de agregación rápida y con las "condensations instantanées" (Maffesoli, 1996). El proceso de construcción, transformación y reconstrucción rápido de las identidades colectivas está 


\section{perifèria}

Número 14, junio 2011

www.periferia.name

relacionado con un lugar y ciertas personas, dentro de una experiencia común que permite la aparición de las identidades colectivas y que, por lo tanto, no son multifocales.

Así que, el concepto de "Belonging" permite la construcción de estas identidades colectivas y de un sentimiento de cohesión y de conjunto cultural (Lovell, 1998) que produce una memoria colectiva local gracias a un proceso histórico. Esta memoria colectiva servirá también de punto de referencia y de agregación entre los individuos, ya que contiene los elementos descritos anteriormente, como las características de la roca y de los bloques o el estilo de escalada propio a esta zona.

"Yet Belonging is also fundamentally defined through a sense of experience, a phenomenology of locality which serves to create, mould and reflect ideals surrounding places" (Lovell, $1998: 1$ )

\section{«Ambiance esthétique » $y \ll$ condensations instantanées 》}

Exceptuando el hecho de que esta categoría de escaladores tienen la misma práctica, o más bien practican una actividad de la misma manera, el elemento de conjunto entre ellos, el que permite la creación tan rápida de relaciones sociales perdurables, surge debido a las condiciones de vida impuestas por este modo de vida. Si el hecho de descubrir otros países, culturas, idiomas y sitios es uno de los motivos clave frente a la adopción de este modo de vida, las condiciones especiales en las cuales se desarrolla la actividad han sido conceptualizadas por Maffesoli (1996) como el ambiente estético ${ }^{4}$.

Entonces, ¿cómo explicar y justificar estos fenómenos de agregación tan rápido entre escaladores de orígenes socio-económicos y culturales tan diversos? Sin tener en cuenta la supuesta barrera lingüística, el hecho de compartir ciertas referencias propias a la práctica representa, por ejemplo, una de las claves que permiten la justificación de estas características, de estas capacidades de agregación tan rápidas. Y no sólo comparten referencias, sino que también valores y símbolos, así como un lenguaje peculiar en el cual cada término se ve asociado a emociones y

\footnotetext{
4 "Ambiance Esthétique" (Maffesoli, $1996: 98$ )
} 


\section{perifèria}

Número 14, junio 2011

www.periferia.name

sensaciones, a una estética y una impresión corporal dependiendo de las capacidades y habilidades de cada uno.

No sé, imola! Es como cuando encuentras a la gente que está metida en este mundo de la escalada, y después ya las [vías] tienes montadas, en sitios como Rodellar y tal, vas a un sitio y vas a otro sitio, isiempre están por ahí! Y es como, lo que ponía el tema, el sigue dándole, no sé si escalas un poco más o menos, también da igual pero el rollo sigue! Es como con los entrenamientos, creo que era un poco para que la gente se pusiera más fuerte en plan...sino crear fanatismo en la zona! Un poco de ambiente, de ánimo! [...]como siempre que vas a escalar, vuelves con el móvil de alguien sabes...de alguien que parece que conoces como si fuera tu hermano casi y al mismo tiempo no sabes nada de él...en plan de mañana te llamo, vamos a no sé que sitio, a hacer no se cual bloque, y tal y cual! Cuando ves un coche y que llegas al sector sabes que esa persona esta ahí, que otra esta ahí, y cuando llegas es como si no tuvieras que buscar a nadie, sabes! Están todos sueltos por todos lados! (Julio, 24 años, España)

Explicar estas agregaciones gracias a la estética, es decir "la faculté commune de sentir et d'éprouver" (Maffesoli, 1996: 98) ha sido el trabajo de Maffesoli gracias a la producción del concepto introducido previamente, "ambiance esthétique", desarrollado en sitios donde el ambiente permite la creación de estas "condensations instantanées" (Maffesoli, 1996: 99).

C'est au sein d'une telle ambiance que s'opèrent des condensations instantanées, fragiles, mais qui dans le moment sont l'objet $d$ 'un fort investissement émotionnel. C'est cet aspect séquentiel qui permet de dépassement du principe d'individuation. (Maffesoli, 1996: 98).

Y esa espontaneidad en la creación y el desarrollo de las relaciones sociales es una de las claves para entender la elección de este modo de vida como una compensación social fundada en el momento, el presente. 


\section{perifèria}

Número 14, junio 2011

www.periferia.name

\section{Por una utilización de la antropología multi-situada}

Utilizar la etnografía multi-situada en el estudio de prácticas deportivas pasionales transformadas en modo de vida ("lifestyle sport") ${ }^{5}$, es decir, en una práctica cotidiana y total, se revela como un instrumento indispensable teniendo en cuenta las características propias de estas prácticas. En nuestro caso, frente a una población móvil dependiente de factores inestables como el clima, que ya ha sido desarrollado con anterioridad, requiere una etnografía flexible y atenta a su objeto. Por eso, es importante replantearse la antropología multi-situada en relación con esas condiciones y desarrollarla de manera útil y práctica, sin decir funcional.

Si la antropología multi-situada ha sido formalizada de mano de Marcus en 1995, no se puede decir que su utilización sea corriente en los estudios antropológicos (Reynoso, 1998). Por varios motivos, la técnica de investigación se ha visto reducida a pocos campos tal como los movimientos transnacionales, por ejemplo, el "translocal" como lo llama Hannerz (2003), elemento central en el estudio de los fenómenos de inmigración.

Aunque se ha utilizado el multi-sitio en varios trabajos de campo, como una manera infinita de comparación ${ }^{6}$, otros lo han utilizado para estudiar campos específicos que necesitaban técnicas de investigación diferentes, más adecuadas a las características del campo, como Sayeux (2008) o Hetherington (2000) por ejemplo. Esta tendencia ha sido resumida de manera explícita por Hannerz: "Obviously the people we are concerned with in the present day field studies tend mostly to be less dependent on seasons and their cycles of activity - on planting and harvesting, or on moving herds to greener pastures" (Hannerz, 2003: 209) y permite una apertura en las posibilidades de utilización del trabajo multi-sitio.

Existen diferentes críticas a la antropología multi-situada ${ }^{7}$, una de ellas es que la limitación de la variable tiempo puede afectar considerablemente al trabajo

\footnotetext{
${ }^{5}$ Véase a Wheaton, 2004

${ }^{6}$ Desde Malinowski hasta los estudios de Hannerz, Wulff o Garsten por ejemplo.

${ }^{7}$ Véase a Reynoso, 1998
} 


\section{perifèria}

Número 14, junio 2011

www.periferia.name

etnográfico. Siguiendo los criterios del trabajo de campo etnográfico de Malinowski (1992), es decir, trabajos de campo de larga estancia, no se pueden establecer relaciones que permitan la recogida de datos fiables, puesto que los criterios de fiabilidad $^{8}$ no han sido formalizados $y$, las etnografías de larga estancia son bastante raras en estos últimos años. Quizás sería adecuado replantearse quien está haciendo actualmente antropología y etnografía.

Parece más productivo tener esto en cuenta que negarlo, mejor jugar con las exigencias actuales para seguir produciendo etnografías y datos de calidad frente a las variedades de trabajos de campo actual. Así que, una antropología multi-situada no siempre será defectuosa por la carencia de tiempo que se impone, ni por las características propias que aceleran el proceso de investigación. Maffesoli ha permitido la formalización de fenómenos complejos y peculiares que, a veces, apoyan cuestiones metodologías como en nuestro caso. Fenómenos que, relacionados con otras características propias al campo, toman toda su relevancia.

\section{Conclusión}

En resumen, más que plantear afirmaciones sobre el instrumento del multi-sitio en la etnografía, el objetivo de este artículo ha sido relacionar una práctica contemporánea, la escalada, desarrollada de forma peculiar y restrictiva por su adopción como modo de vida, con la teoría antropológica del espacio y del paisaje, como una introducción sencilla a una construcción social compleja. En el ámbito de las Leisure Studies, su examen a través del concepto de modo de vida relacionado con su medio ambiente, nunca había sido examinado, aunque su rol es determinante por los motivos que dirigen a los aficionados a volverse fanáticos y a enfocar su vida alrededor de una sola práctica. A día de hoy, el número de escaladores se va ampliando rápidamente en un contexto general de desarrollo avanzado de los lifestyle sports y sus relaciones con el medio ambiente constituye su entorno de realización requiriendo atención, tanto a nivel de la escalada, como a nivel de un marco más amplio perteneciente a la socio-antropología del espacio. En

\footnotetext{
${ }^{8}$ Véase a Latour, 2001
} 


\section{perifèria}

Número 14, junio 2011

www.periferia.name

el mundo, y especialmente en España, donde se localizan una multitud de zonas de escalada, se han creado varios tipos de "conflictos" entre diferentes tipos de usuarios del espacio y los poderes públicos y privados, dando nacimiento a fenómenos nuevos que requieren atención y que pueden ser desarrollados en un ámbito más amplio de las ciencias sociales.

Por otra parte, los fenómenos de agregación rápida, formalizados en otros contextos por Maffesoli, son para mí unas de las claves para el trabajo etnográfico actual, en un mundo social cambiante relacionado con la antropología multi-situada que toman gran importancia, aunque estos aspectos pueden parecer imperceptibles. Además, estas concepciones y usos del espacio, su semiótica y su simbólica pueden ser reveladoras de tendencias de larga escala, reveladoras de dinámicas actuales amplias, aunque siguen refiriéndose a poblaciones limitadas y que no obstante, y teniendo en cuenta el tamaño de estos fenómenos, ciertos autores podrían llamar "marginalité massive" (De Certeau, 1990: XLIII).

\section{Bibliografía citada}

Aubel, Olivier (2005). L'escalade libre en France. Paris: I'harmattan.

Appadurai, Arjun (1991). Global Ethnoscapes: Notes and Querries for an Transnational Anthropology. En R.G. Fox (Ed.) Interventions: Anthropology of the Present. Sante Fe: School of American Research, pp.191-210.

Beal, Becky; Wilson, Charlene (2004). "Chicks dig scars: commercialisation and the transformations of skateboarder' identities". En Wheaton, Belinda. Understanding Lifestyle sports: consumption, identity and difference. Routledge: London, pp.3155.

Bourdieu, Pierre (1994). Raisons pratiques: Sur la théorie de l'action. Paris: Editions du Seuil.

De Certau, Michel, (1990 [1980]). L'invention du quotidien. Tome 1 : Les arts de faire. Paris : Gallimard.

De Leseleuc, Eric (2004). Les voleurs de falaise: un territoire d'escalade entre espace public et espace privé. Pessac: Maison des sciences de I'homme 


\section{perifèria}

Número 14, junio 2011

www.periferia.name

d'Aquitaine.

Descola, Philippe (2005). Par delà nature et culture. Paris : Editions Gallimard.

Dumont, Guillaume (2010). De l'escalade au voyage. Ethnographie d'un mode de vie. Mémoire de Master en anthropologie à finalité approfondie, Université Libre de Bruxelles.

Donnelly, Michele (2006). "Studying Extreme Sports: Beyond the Core Participants". Journal of Sport and Social Issues, 30 (219), pp.219-224.

Donnelly, Peter; Young, Kevin (1988) "The construction and confirmation of Identity in Sport Subcultures", Sociology of Sport Journal, 5, pp.223-240.

Garsten, Christina (1994). Apple World. Stockholm Studies in Social Anthropology 33. Stockholm: Almqyist and Wiksell International.

Hannerz, UIf (1996). Transational connections: culture, people, places. London: Routledge.

(2003) "Being there...and there...and there! Reflections on Multi-Site Ethnography". Ethnography, 4, pp.201-216.

Hetherington, Kevin (2000). New age travellers: vanloads of uproarious humanity. New-York: Cassel.

Hirsch, Eric; O'Hanlon, Michael (Ed) (1995). The Anthropology of Landscape: Perspectives on Place and Space. Oxford: Clarendon Press

Latour, Bruno (2001). «Sol Amazonien et circulation de la référence ». En : L'espoir de Pandore. Pour une version réaliste de l'activité scientifique. pp.33-81. Paris : La Découverte.

Lefebvre, Henri (1974). La production de l'Espace. Paris : Editions Anthropos. Lovell, Nadia (1998). Locality and Belonging. New-York: Routledge. Maffesoli, Michel (1988). Le temps des tribus : le déclin de l'individualisme dans les sociétés de masse. Paris : Méridiens Klincksieck. (1996). Du nomadisme: vagabondages initiatiques. Paris: Table Ronde. 


\section{perifèria}

Número 14, junio 2011

www.periferia.name

Malinowski, Bronislaw (1993 [1922]). Les Argonautes du Pacifique occidental. Paris: Gallimard.

Marcus, Georges (1995). "Ethnography in/of the World System: The Emergence of Multi-Sited Ethnography", Annual Review of Anthropology, 24, pp.95-117.

Merleau-Ponty, Maurice (1985 [1944]). Fenomenología de la percepción. Barcelona: Planeta Agostini.

Reynoso, Carlos (1998). Corrientes en antropología contemporánea. Universidad de Buenos Aires: Biblos.

Sayeux, Anne-Sophie (2008). Surfeurs, l'être au monde. Une analyse socioanthropologique. Rennes: Presses Universitaires de Rennes.

(2010). «Les paysages vagues ». En Sirost, Olivier. Paysages sensibles. Société, 109, pp.91-103.

Soule, Bastien; Walk, Steve (2007). « Comment rester "alternatif"? Sociologie des pratiquants sportifs en quête d'authenticité subculturelle ». Corps, 1 (2), pp.67-72.

Stebbins, Robert, (1992). Amateurs, Professionals, and Serious leisure. Beverly Hill: Sage.

Thornton, Sarah (1997). The social logic of subcultural capital. En: Thornton, Sarah; Gelder, Ken. The subcultures reader. London: Routledge, pp. 200-212.

Wacquant, Loic (2004). Entre las cuerdas. Cuadernos etnográficos de un aprendiz de boxeador. Madrid: Alianza.

Wheaton, Belinda (2000). "Just do it: Consuption, commitment and identity in the windsurfing culture", Sociology of Sport Journal, 17(3), pp. 254-274.

; Beal, Becky (2003). "Keeping it real: Subcultural media and the discourses of authenticity in alternative sport", International Review for the Sociology of Sport, 38, pp.155-176.

(2004). Understanding Lifestyle sports: consumption, identity and difference. Routledge: London. 


\title{
perifèria
}

\author{
Número 14, junio 2011 \\ www.periferia.name
}

Wulff, Helen (2000). "Access to a closed world: methods for a multilocale study on ballet as a career". En Veret Amit (ed). Constructing the field: ethnographic fieldwork in the contemporary world. London: Routledge, pp. 147-161. 